\title{
A dissertation on tooth size and arch dimension in uncrowded versus crowded Class I malocclusion
}

\section{Jahan $\mathrm{H}^{1}$ BDS, FCPS and Hossain $M \mathrm{Z}^{2}$ BDS, PhD,}

\section{ABSTRACT}

Aim: The purpose of this investigation was to examine the extent to which arch dimension or tooth size contributes to dental crowding.

Materials and Methods: Two groups of dental casts were selected. Each group consisted of 30 pairs of dental casts including equal male and female samples. The first group had Class I malocclusion without crowding. The second group exhibited Class I malocclusion with dental crowding (more than $5 \mathrm{~mm}$ space deficiency). The following parameters were measured and used to compare the two groups: individual and collective mesiodistal widths of tooth, dental arch length, as well as buccal and lingual dental arch widths in the canine and molar regions.

Results: Statistically significant differences in both tooth widths and transverse arch dimensions (widths) were found between the crowded and the normal groups. The crowded group was found to have a significantly smaller maxillary arch width and larger tooth size when compared with the uncrowded or normal group. There were no significant differences in arch length in the two groups in either the maxilla or the mandible. In comparing the anterior and overall Bolton ratios no significant difference was detected between the crowded and normal groups.

Conclusion: The results of this study suggest under under two study groups (Class I skeletal base of crowded and uncrowded group) tooth size has a greater role in developing dental crowding. (Ban J Orthod and Dentofac Orthop, Oct. 2011; Vol-2, No. 1, p 1-6

\section{INTRODUCTION}

Malocclusion is a developmental condition. In most instances, malocclusion is caused, not by some pathologic process, but by moderate distortions of normal development. ${ }^{1}$ Malocclusion is one of the commonest aesthetic and functional problems in our country. The most common type of malocclusion observed in both mixed dentition and permanent dentition patients is crowding. These patients are usually referred to the orthodontist by the family dentist or by the patient's parents because of obvious dental irregularities or lack of sufficient space for tooth eruption. Such patients usually present with a Class I molar relationship or a tendency toward either Class II or Class III malocclusion. ${ }^{2}$

Crowding of the teeth, the most common type of malocclusion at present undoubtedly is related in part to the continuing reduction in jaw size in human evolutionary development. Jaw dimension do seem to have a strong genetic control, and the transverse dimensions directly affect the amount of space for the teeth. ${ }^{1}$

A relative harmony in the mesiodistal dimension of the maxillary and mandibular teeth is a major factor in coordinating posterior interdigitation, overbite and overjet in centric occlusion. ${ }^{3}$

Tooth size must also be in harmony with arch size to allow proper alignment. ${ }^{4}$ A significant variation in this harmony will lead to malocclusion and difficulties in obtaining an occlusion with optimal overjet, overbite and Class I canine and molar relationships.

Dental crowding is the consequence of a tooth size and dental arch dimension discrepancy. Identifying the etiology of malocclusion has proven to be one of the most important issues in orthodontics. Different theories have tried to explain the etiology of dental crowding including hereditary and environmental factors. ${ }^{5}$ Thus this type of study will be helpful in proper diagnosis and treatment planning prior to orthodontic treatment.

Tooth size greatly differs among different races. ${ }^{6}$ In an investigation of tooth size of 139 Swedish boys in 1951, Lundstrom ${ }^{7}$ found crowding was greater in those individuals with larger teeth. The results of Fastlicht's ${ }^{8}$ studies were consistent with Lundstrom's and revealed a significant relationship between tooth size and crowding. In another study, Doris et al. ${ }^{9}$ found larger tooth sizes in crowded cases rather than in the non-crowded cases, with the greatest difference found between the maxillary lateral incisor and second premolars. In an investigation performed by Howe et al. ${ }^{10}$ comparisons were made between crowded and non-crowded groups using study models. They indicated that arch dimension made a greater contribution to dental crowding than tooth size. Other investigations found the same correlation between arch dimensions and dental crowding. ${ }^{11-13}$

${ }^{1}$ Lecturer, Department of Orthodontics and Dentofacial Orthopedics, Dhaka Dental College. ${ }^{2}$ Professor and Head, Department of Orthodontics and Dentofacial Orthopedics, Dhaka Dental College. 


\section{Rational of the study}

In our country the incidence of Class I malocclusion is $55.22 \%$ among 111 patients $^{14}$ and another study revealed $33.8 \%$ among 222 patients. ${ }^{15}$ No elaborate study has so far been made over tooth size and arch dimension in uncrowded versus crowded Class I malocclusions in our country. This study will help orthodontist to establish a appropriate treatment plan. Besides the patients/parents satisfaction could be anticipated. This study will also generate interest for further research in this field.

\section{HYPOTHESIS}

Tooth size and arch dimension have a greater role in developing malocclusion.

\section{MATERIALS AND METHODS}

\section{Sample selection}

In this study, 60 pairs of study models were selected from the patients and students of the Orthodontics and Dentofacial Orthopedics Department of Dhaka Dental College and Hospital and were divided into two groups. The first group consisted of 30 pairs of study models of Class I occlusions based on a Class I skeletal relationship without any abnormal spacing or crowding. The samples were divided evenly between the sexes ( 15 females and 15 males). They all exhibited a straight profile, normal overbite and overjet and Class I canine and molar relationships. The second group consisted of 30 pairs of study models of Class I malocclusions with a Class I skeletal base which had more than $5 \mathrm{~mm}$ of dental crowding. Again, the samples were equally divided between the sexes (15 females and 15 males).

\section{Selection criteria}

\section{Inclusion Criteria}

1. Permanent dentition with no missing or supernumerary teeth (excluding third molars).

2. No proximal restorations.

3. No previous orthodontic treatment.

\section{Exclusion criteria}

1. Non co-operative patient.

2. Patient with systemic diseases.

3. Missing or supernumerary teeth.

4. Previous orthodontic treatment.

5. Proximal restoration.

\section{Measurement}

The following measurements were recorded:

1. The largest mesiodistal width of each tooth (except the second and third molars) on each arch.

2. Buccal and lingual inter-canine width

3. Buccal and lingual inter-molar width

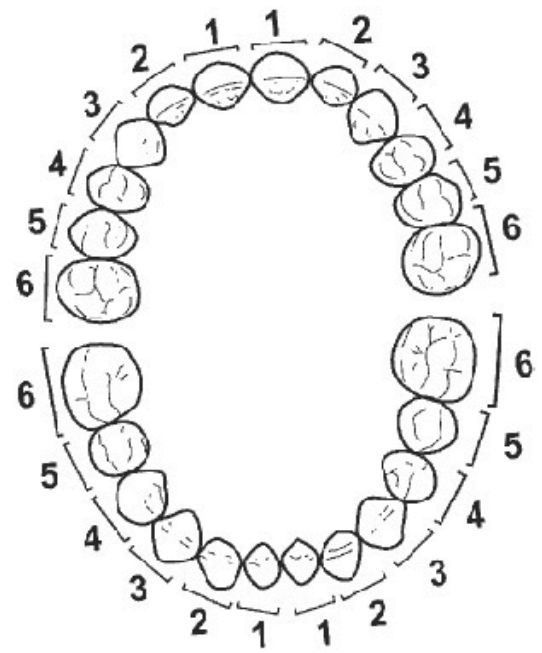

Fig-1 The mesiodistal widths of the teeth

The mesiodistal widths of the teeth were measured in the largest area.

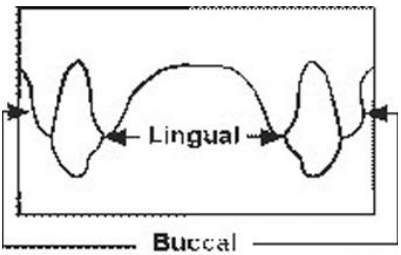

Fig-2. Buccal and lingual intercanine width.

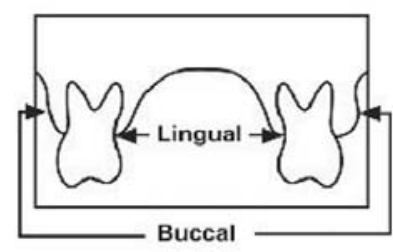

Fig-3.Buccal and lingual intermolar width.
Arch dimensions in the canine and molar regions were measured buccally and lingually. The buccal arch dimension was measured $5 \mathrm{~mm}$ apical to the mesiodistal centre of the gingival margin of the canine tooth on one side to the same point on the contralateral side.

On the lingual side, the distance between midpoints on the cervical region of the canine in one side was measured to the corresponding point on the contra-lateral side. The same procedure was performed in the molar region.

\section{RESULTS}

This study was an analytical and comparative study conducted among the 60 patients and students of Dhaka Dental College and Hospital. The occlusion of these patients was class- 1 crowded and normal occlusion (Class I uncrowded). Tooth size and arch dimension were calculated for each individual models of Angle's Class I crowded and the corresponding normal occlusion (Class I uncrowded) groups under study. The statistical tests to be used for analysis of data was t- test. In this analytical test the level of significance $p$ value $<0.05$ was considered significant.

2

Bangladesh Journal of Orthodontics and Dentofacial Orthopedics (BJO and DFO)

Vol. 2, No. 1, October 2011 
A dissertation on tooth size and arch dimension in uncrowded versus crowded Class I malocclusion.

Table-I:Collective mesiodistal width of right and left maxillary teeth for each sex in crowded and normal occlusion (mm)

\begin{tabular}{|c|c|c|c|c|c|c|c|c|c|}
\hline \multirow[b]{2}{*}{$\begin{array}{l}\text { Name of the } \\
\text { teeth }\end{array}$} & \multicolumn{3}{|c|}{ Male $(n=30)$} & \multicolumn{3}{|c|}{ Female $(n=30)$} & \multicolumn{3}{|c|}{ Total $(n=60)$} \\
\hline & $\begin{array}{l}\begin{array}{l}\text { Normal } \\
(\mathrm{n}=15)\end{array} \\
\text { Mean } \\
( \pm \mathrm{SD})\end{array}$ & $\begin{array}{l}\text { Crowded } \\
(\mathrm{n}=15) \\
\text { Mean } \\
( \pm \mathrm{SD})\end{array}$ & $P$ value & $\begin{array}{l}\text { Normal } \\
(\mathrm{n}=15) \\
\text { Mean } \\
( \pm \mathrm{SD})\end{array}$ & $\begin{array}{l}\text { Crowded } \\
(\mathrm{n}=15) \\
\text { Mean } \\
( \pm \mathrm{SD})\end{array}$ & $\mathrm{P}$ value & $\begin{array}{l}\text { Normal } \\
(\mathrm{n}=30) \\
\text { Mean } \\
( \pm \text { SD })\end{array}$ & $\begin{array}{l}\text { Crowded } \\
(\mathrm{n}=30) \\
\text { Mean } \\
( \pm \mathrm{SD})\end{array}$ & $\mathrm{P}$ value \\
\hline $\begin{array}{l}\text { Max.Central } \\
\text { incisors }\end{array}$ & $\begin{array}{l}8.54 \\
( \pm 0.48)\end{array}$ & $\begin{array}{l}8.40 \\
( \pm 2.21)\end{array}$ & $0.82 \mathrm{NS}$ & $\begin{array}{l}8.45 \\
( \pm 0.58)\end{array}$ & $\begin{array}{l}8.68 \\
( \pm 1.40)\end{array}$ & $0.57 \mathrm{NS}$ & $\begin{array}{l}8.49 \\
( \pm 0.52)\end{array}$ & $\begin{array}{l}8.54 \\
( \pm 1.82)\end{array}$ & $0.89 \mathrm{NS}$ \\
\hline $\begin{array}{l}\text { Max. Lateral } \\
\text { incisors }\end{array}$ & $\begin{array}{l}6.66 \\
( \pm 0.72)\end{array}$ & $\begin{array}{l}7.71 \\
( \pm 0.59)\end{array}$ & $<0.001 * * *$ & $\begin{array}{l}6.67 \\
( \pm 0.59)\end{array}$ & $\begin{array}{l}7.81 \\
( \pm 0.51)\end{array}$ & $<0.001 * * *$ & $\begin{array}{l}6.66 \\
( \pm 0.65)\end{array}$ & $\begin{array}{l}7.76 \\
( \pm 0.54)\end{array}$ & $<0.001 * * *$ \\
\hline $\begin{array}{l}\text { Max. } \\
\text { Canines }\end{array}$ & $\begin{array}{l}7.73 \\
( \pm 0.55)\end{array}$ & $\begin{array}{l}8.28 \\
( \pm 0.58)\end{array}$ & $0.01 *$ & $\begin{array}{l}7.42 \\
( \pm 0.40)\end{array}$ & $\begin{array}{l}8.27 \\
( \pm 0.50)\end{array}$ & $<0.001 * * *$ & $\begin{array}{l}7.58 \\
( \pm 0.50)\end{array}$ & $\begin{array}{l}8.27 \\
( \pm 0.53)\end{array}$ & $<0.001 * * *$ \\
\hline $\begin{array}{l}\text { Max. 1st } \\
\text { Premolars }\end{array}$ & $\begin{array}{l}7.03 \\
( \pm 0.32)\end{array}$ & $\begin{array}{l}7.51 \\
( \pm 0.34)\end{array}$ & $0.001 * * *$ & $\begin{array}{l}7.14 \\
( \pm 0.34)\end{array}$ & $\begin{array}{l}7.48 \\
( \pm 0.35)\end{array}$ & $0.01 *$ & $\begin{array}{l}7.08 \\
( \pm 0.33)\end{array}$ & $\begin{array}{l}7.49 \\
( \pm 0.34)\end{array}$ & $<0.001 * * *$ \\
\hline $\begin{array}{l}\text { Max.2nd } \\
\text { Premolars }\end{array}$ & $\begin{array}{l}6.57 \\
( \pm 0.50)\end{array}$ & $\begin{array}{l}6.97 \\
( \pm 0.44)\end{array}$ & $0.02 *$ & $\begin{array}{l}6.60 \\
( \pm 0.46)\end{array}$ & $\begin{array}{l}7.06 \\
( \pm 0.33)\end{array}$ & $0.004 * * *$ & $\begin{array}{l}6.58 \\
( \pm 0.47)\end{array}$ & $\begin{array}{l}7.01 \\
( \pm 0.38)\end{array}$ & $<0.001 * * *$ \\
\hline $\begin{array}{l}\text { Max.1st } \\
\text { Molars }\end{array}$ & $\begin{array}{l}10.26 \\
( \pm 0.47)\end{array}$ & $\begin{array}{l}10.92 \\
( \pm 0.62)\end{array}$ & $0.003 * * *$ & $\begin{array}{l}10.37 \\
( \pm 0.61)\end{array}$ & $\begin{array}{l}10.72 \\
( \pm 0.59)\end{array}$ & $0.11 \mathrm{NS}$ & $\begin{array}{l}10.31 \\
( \pm 0.54)\end{array}$ & $\begin{array}{l}10.82 \\
( \pm 0.60)\end{array}$ & $0.001 * * *$ \\
\hline
\end{tabular}

$\mathrm{NS}=$ Not significant., $* / * * *-\operatorname{Significant}(\mathrm{p}<0.05)$

Table-II Collective mesiodistal widths of right and left mandibular teeth for each sex in crowded and normal occlusion(mm).

\begin{tabular}{|c|c|c|c|c|c|c|c|c|c|}
\hline \multirow[b]{2}{*}{$\begin{array}{l}\text { Name of the } \\
\text { teeth }\end{array}$} & \multicolumn{3}{|c|}{ Male $(n=30)$} & \multicolumn{3}{|c|}{ Female $(n=30)$} & \multicolumn{3}{|c|}{ Total $(n=60)$} \\
\hline & $\begin{array}{l}\text { Normal } \\
(\mathrm{n}=15) \\
\text { Mean } \\
( \pm \mathrm{SD})\end{array}$ & $\begin{array}{l}\text { Crowded } \\
(\mathrm{n}=15) \\
\text { Mean } \\
( \pm \mathrm{SD})\end{array}$ & $P$ value & $\begin{array}{l}\text { Normal } \\
(\mathrm{n}=15) \\
\text { Mean } \\
( \pm \mathrm{SD})\end{array}$ & $\begin{array}{l}\text { Crowded } \\
(\mathrm{n}=15) \\
\text { Mean } \\
( \pm \mathrm{SD})\end{array}$ & $\mathrm{P}$ value & $\begin{array}{l}\text { Normal } \\
(\mathrm{n}=30) \\
\text { Mean } \\
( \pm \mathrm{SD})\end{array}$ & $\begin{array}{l}\text { Crowded } \\
(\mathrm{n}=30) \\
\text { Mean } \\
( \pm \mathrm{SD})\end{array}$ & $P$ value \\
\hline $\begin{array}{l}\text { Mand.Central } \\
\text { incisors }\end{array}$ & $\begin{array}{l}5.40 \\
( \pm 0.37)\end{array}$ & $\begin{array}{l}5.96 \\
( \pm 0.37)\end{array}$ & $<0.001 * * *$ & $\begin{array}{l}5.60 \\
( \pm 0.47)\end{array}$ & $\begin{array}{l}5.95 \\
( \pm 0.44)\end{array}$ & $0.04 *$ & $\begin{array}{l}5.50 \\
( \pm 0.43)\end{array}$ & $\begin{array}{l}5.95 \\
( \pm 0.40)\end{array}$ & $<0.001 * * *$ \\
\hline $\begin{array}{l}\text { Mand. Lateral } \\
\text { incisors }\end{array}$ & $\begin{array}{l}6.06 \\
( \pm 0.26)\end{array}$ & $\begin{array}{l}6.47 \\
( \pm 0.43)\end{array}$ & $0.005 * * *$ & $\begin{array}{l}5.90 \\
( \pm 0.28)\end{array}$ & $\begin{array}{l}6.44 \\
( \pm 0.44)\end{array}$ & $0.001 * * *$ & $\begin{array}{l}5.98 \\
( \pm 0.27)\end{array}$ & $\begin{array}{l}6.46 \\
( \pm 0.43)\end{array}$ & $<0.001 * * *$ \\
\hline $\begin{array}{l}\text { Mand. } \\
\text { Canines }\end{array}$ & $\begin{array}{l}6.76 \\
( \pm 0.41)\end{array}$ & $\begin{array}{l}7.30 \\
( \pm 0.42)\end{array}$ & $0.001 * * *$ & $\begin{array}{l}6.56 \\
( \pm 0.41)\end{array}$ & $\begin{array}{l}7.22 \\
( \pm 0.28)\end{array}$ & $<0.001^{* * *}$ & $\begin{array}{l}6.66 \\
( \pm 0.41)\end{array}$ & $\begin{array}{l}7.26 \\
( \pm 0.35)\end{array}$ & $<0.001 * * *$ \\
\hline $\begin{array}{l}\text { Mand. 1st } \\
\text { Premolars }\end{array}$ & $\begin{array}{l}7.03 \\
( \pm 0.43)\end{array}$ & $\begin{array}{l}7.70 \\
( \pm 0.50)\end{array}$ & $0.001 * * *$ & $\begin{array}{l}6.90 \\
( \pm 0.53)\end{array}$ & $\begin{array}{l}7.56 \\
( \pm 0.39)\end{array}$ & $0.001 * * *$ & $\begin{array}{l}6.96 \\
( \pm 0.48)\end{array}$ & $\begin{array}{l}7.63 \\
( \pm 0.44)\end{array}$ & $<0.001 * * *$ \\
\hline $\begin{array}{l}\text { Mand.2nd } \\
\text { Premolars }\end{array}$ & $\begin{array}{l}6.82 \\
( \pm 0.35)\end{array}$ & $\begin{array}{l}7.44 \\
( \pm 0.45)\end{array}$ & $<0.001 * * *$ & $\begin{array}{l}7.02 \\
( \pm 0.59)\end{array}$ & $\begin{array}{l}7.36 \\
( \pm 0.46)\end{array}$ & $0.09 \mathrm{NS}$ & $\begin{array}{l}6.92 \\
( \pm 0.49)\end{array}$ & $\begin{array}{l}7.40 \\
( \pm 0.45)\end{array}$ & $<0.001 * * *$ \\
\hline $\begin{array}{l}\text { Mand .1st } \\
\text { Molars }\end{array}$ & $\begin{array}{l}10.68 \\
( \pm 0.51)\end{array}$ & $\begin{array}{l}11.36 \\
( \pm 0.51)\end{array}$ & $0.001 * * *$ & $\begin{array}{l}10.79 \\
( \pm 0.61)\end{array}$ & $\begin{array}{l}11.42 \\
( \pm 0.60)\end{array}$ & $0.008 * * *$ & $\begin{array}{l}10.73 \\
( \pm 0.56)\end{array}$ & $\begin{array}{l}11.39 \\
( \pm 0.55)\end{array}$ & $<0.001 * * *$ \\
\hline
\end{tabular}

$\mathrm{NS}=$ Not significant., $\quad * / * * *-$ Significant $(\mathrm{p}<0.05)$ 
Table -III Sum of six (6) maxillary anterior teeth for each sex in crowded and normal occlusion (mm).

\begin{tabular}{|c|c|c|c|c|c|c|c|c|c|}
\hline \multirow[b]{2}{*}{ Region } & \multicolumn{3}{|c|}{ Male $(n=30)$} & \multicolumn{3}{|c|}{ Female $(n=30)$} & \multicolumn{3}{|c|}{ Total $(n=60)$} \\
\hline & $\begin{array}{l}\text { Normal } \\
(\mathrm{n}=15) \\
\text { Mean } \\
( \pm \mathrm{SD})\end{array}$ & $\begin{array}{l}\text { Crowded } \\
(\mathrm{n}=15) \\
\text { Mean } \\
( \pm \mathrm{SD})\end{array}$ & $P$ value & $\begin{array}{l}\text { Normal } \\
(\mathrm{n}=15) \\
\text { Mean } \\
( \pm \mathrm{SD})\end{array}$ & $\begin{array}{l}\text { Crowded } \\
(\mathrm{n}=15) \\
\text { Mean } \\
( \pm \mathrm{SD})\end{array}$ & $P$ value & $\begin{array}{l}\text { Normal } \\
(\mathrm{n}=30) \\
\text { Mean } \\
( \pm \mathrm{SD})\end{array}$ & $\begin{array}{l}\text { Crowded } \\
(\mathrm{n}=30) \\
\text { Mean } \\
( \pm \mathrm{SD})\end{array}$ & $P$ value \\
\hline $\begin{array}{l}\text { Sum of max- } \\
\text { illary } 6 \\
\text { anteriors }\end{array}$ & $\begin{array}{l}45.72 \\
( \pm 3.10)\end{array}$ & $\begin{array}{l}48.65 \\
( \pm 3.90)\end{array}$ & $0.03 *$ & $\begin{array}{l}44.97 \\
( \pm 2.59)\end{array}$ & $\begin{array}{l}49.38 \\
( \pm 2.95)\end{array}$ & $<0.001 * * *$ & $\begin{array}{l}45.35 \\
( \pm 2.83)\end{array}$ & $\begin{array}{l}49.01 \\
( \pm 3.42)\end{array}$ & $<0.001 * * *$ \\
\hline
\end{tabular}

$\mathrm{NS}=$ Not significant., $* / * * *-$ Significant $(\mathrm{p}<0.05)$

Table -IV Sum of six (6) mandibular anterior teeth for each sex in crowded and normal occlusion (mm).

\begin{tabular}{|c|c|c|c|c|c|c|c|c|c|}
\hline \multirow[b]{2}{*}{ Region } & \multicolumn{3}{|c|}{ Male $(n=30)$} & \multicolumn{3}{|c|}{ Female $(n=30)$} & \multicolumn{3}{|c|}{ Total $(n=60)$} \\
\hline & $\begin{array}{l}\text { Normal } \\
(\mathrm{n}=15) \\
\text { Mean } \\
( \pm \mathrm{SD})\end{array}$ & $\begin{array}{l}\text { Crowded } \\
(\mathrm{n}=15) \\
\text { Mean } \\
( \pm \mathrm{SD})\end{array}$ & $P$ value & $\begin{array}{l}\text { Normal } \\
(\mathrm{n}=15) \\
\text { Mean } \\
( \pm \mathrm{SD})\end{array}$ & $\begin{array}{l}\text { Crowded } \\
(\mathrm{n}=15) \\
\text { Mean } \\
( \pm \mathrm{SD})\end{array}$ & $P$ value & $\begin{array}{l}\text { Normal } \\
(\mathrm{n}=30) \\
\text { Mean } \\
( \pm \mathrm{SD})\end{array}$ & $\begin{array}{l}\text { Crowded } \\
(\mathrm{n}=30) \\
\text { Mean } \\
( \pm \mathrm{SD})\end{array}$ & $\mathrm{P}$ value \\
\hline $\begin{array}{l}\text { Sum of } \\
\text { mandibular } \\
6 \text { anteriors }\end{array}$ & $\begin{array}{l}36.31 \\
( \pm 1.83)\end{array}$ & $\begin{array}{l}39.34 \\
( \pm 2.10)\end{array}$ & $<0.001 * * *$ & $\begin{array}{l}36.02 \\
( \pm 1.87)\end{array}$ & $\begin{array}{l}39.12 \\
( \pm 2.09)\end{array}$ & $<0.001 * * *$ & $\begin{array}{l}36.16 \\
( \pm 1.82)\end{array}$ & $\begin{array}{l}39.23 \\
( \pm 2.06)\end{array}$ & $<0.001 * * *$ \\
\hline
\end{tabular}

$\mathrm{NS}=$ Not significant., $\quad * / * * *$ - Significant $(\mathrm{p}<0.05)$

Table -V Sum of twelve (12) maxillary teeth for each sex in crowded and normal occlusion (mm).

\begin{tabular}{|c|c|c|c|c|c|c|c|c|c|}
\hline \multirow[b]{2}{*}{ Region } & \multicolumn{3}{|c|}{ Male $(n=30)$} & \multicolumn{3}{|c|}{ Female $(n=30)$} & \multicolumn{3}{|c|}{ Total $(n=60)$} \\
\hline & $\begin{array}{l}\text { Normal } \\
(\mathrm{n}=15) \\
\text { Mean } \\
( \pm \mathrm{SD})\end{array}$ & $\begin{array}{l}\text { Crowded } \\
(\mathrm{n}=15) \\
\text { Mean } \\
( \pm \mathrm{SD})\end{array}$ & $\mathrm{P}$ value & $\begin{array}{l}\text { Normal } \\
(\mathrm{n}=15) \\
\text { Mean } \\
( \pm \mathrm{SD})\end{array}$ & $\begin{array}{l}\text { Crowded } \\
(\mathrm{n}=15) \\
\text { Mean } \\
( \pm \mathrm{SD})\end{array}$ & $\mathrm{P}$ value & $\begin{array}{l}\text { Normal } \\
(\mathrm{n}=30) \\
\text { Mean } \\
( \pm \mathrm{SD})\end{array}$ & $\begin{array}{l}\text { Crowded } \\
(\mathrm{n}=30) \\
\text { Mean } \\
( \pm \mathrm{SD})\end{array}$ & $\mathrm{P}$ value \\
\hline $\begin{array}{l}\text { Sum of } 12 \\
\text { maxillary } \\
\text { teeth }\end{array}$ & $\begin{array}{l}93.40 \\
( \pm 4.76)\end{array}$ & $\begin{array}{l}101.42 \\
( \pm 4.93)\end{array}$ & $<0.001 * * *$ & $\begin{array}{l}93.18 \\
( \pm 4.47)\end{array}$ & $\begin{array}{l}100.75 \\
( \pm 3.52)\end{array}$ & $<0.001 * * *$ & $\begin{array}{l}93.29 \\
( \pm 4.54)\end{array}$ & $\begin{array}{l}101.08 \\
( \pm 4.23)\end{array}$ & $<0.001 * * *$ \\
\hline
\end{tabular}

$\mathrm{NS}=$ Not significant., $\quad * / * * *-$ Significant $(\mathrm{p}<0.05)$

Table -VI Sum of twelve (12) mandibular teeth for each sex in crowded and normal occlusion (mm).

\begin{tabular}{|c|c|c|c|c|c|c|c|c|c|}
\hline \multirow[b]{2}{*}{ Region } & \multicolumn{3}{|c|}{ Male $(n=30)$} & \multicolumn{3}{|c|}{ Female $(n=30)$} & \multicolumn{3}{|c|}{ Total $(n=60)$} \\
\hline & $\begin{array}{l}\begin{array}{l}\text { Normal } \\
(\mathrm{n}=15)\end{array} \\
\text { Mean } \\
( \pm \mathrm{SD})\end{array}$ & $\begin{array}{l}\text { Crowded } \\
(\mathrm{n}=15) \\
\text { Mean } \\
( \pm \text { SD })\end{array}$ & $P$ value & $\begin{array}{l}\text { Normal } \\
(\mathrm{n}=15) \\
\text { Mean } \\
( \pm \text { SD })\end{array}$ & $\begin{array}{l}\begin{array}{l}\text { Crowded } \\
(\mathrm{n}=15)\end{array} \\
\text { Mean } \\
( \pm \text { SD })\end{array}$ & $\mathrm{P}$ value & $\begin{array}{l}\text { Normal } \\
(\mathrm{n}=30) \\
\text { Mean } \\
( \pm \mathrm{SD})\end{array}$ & $\begin{array}{l}\text { Crowded } \\
(\mathrm{n}=30) \\
\text { Mean } \\
( \pm \mathrm{SD})\end{array}$ & $\mathrm{P}$ value \\
\hline $\begin{array}{l}\text { Sum of } 12 \\
\text { mandibular } \\
\text { teeth }\end{array}$ & $\begin{array}{l}85.29 \\
( \pm 3.56)\end{array}$ & $\begin{array}{l}92.26 \\
( \pm 3.87)\end{array}$ & $<0.001 * * *$ & $\begin{array}{l}85.20 \\
( \pm 4.63)\end{array}$ & $\begin{array}{l}91.66 \\
( \pm 3.49)\end{array}$ & $<0.001 * * *$ & $\begin{array}{l}85.24 \\
( \pm 4.06)\end{array}$ & $\begin{array}{l}91.96 \\
( \pm 3.63)\end{array}$ & $<0.001 * * *$ \\
\hline
\end{tabular}

$\mathrm{NS}=$ Not significant., $* / * * *$ - Significant $(\mathrm{p}<0.05)$

4 
Maxillary arch dimensions (widths) for each sex in crowded and normal occlusion (mm) showing in the bar chart (fig-12).
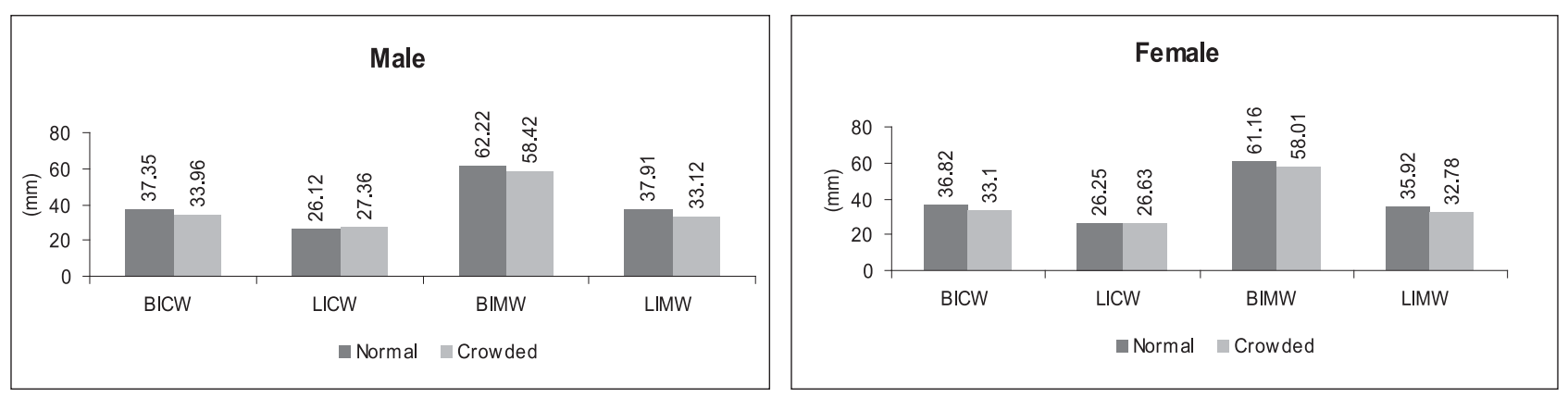

$\mathrm{BICW}=$ Buccal inter canine width, $\mathrm{LICW}=$ Lingual inter-canine width, $\mathrm{BIMW}=$ Buccal inter molar width, $\mathrm{LIMW}=$ Lingual inter molar width

Fig- 3. Maxillary arch dimensions (widths) for each sex in crowded and normal occlusion (mm)

Mandibular arch dimensions (widths) for each sex in crowded and normal occlusion (mm) showing in the bar chart (fig- 4).
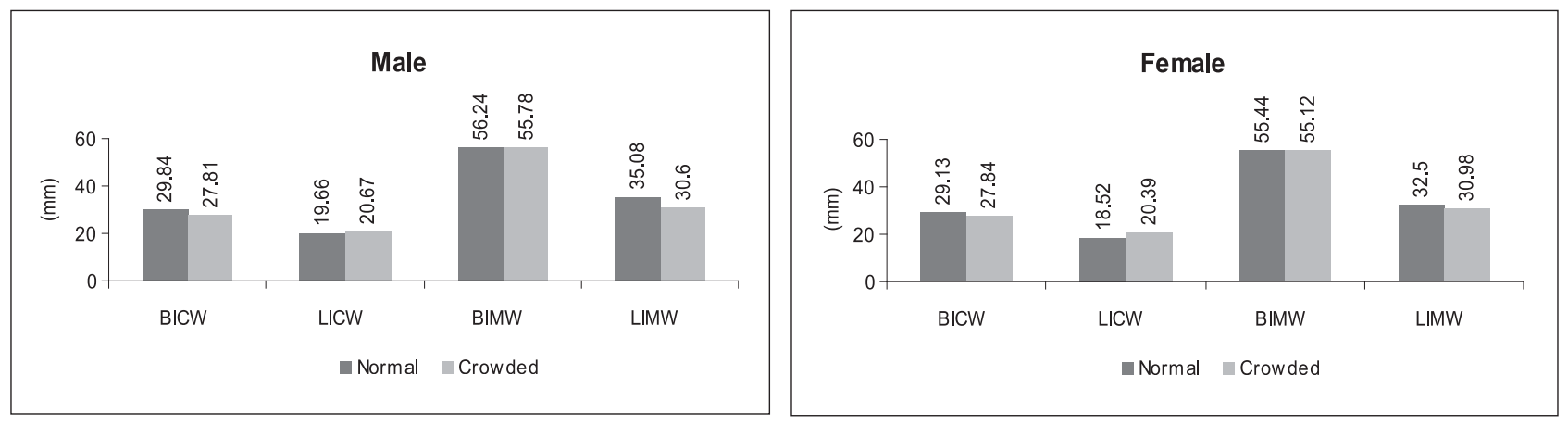

$\mathrm{BICW}=$ Buccal inter canine width, LICW= Lingual inter-canine width, BIMW= Buccal inter molar width, LIMW= Lingual inter molar width

Fig- 4. Mandibular arch dimensions (widths) for each sex in crowded and normal occlusion (mm)

\section{DISCUSSION}

This analytical and comparative study was conducted in the department of Orthodontics and Dentofacial Orthopedics at Dhaka Dental College and Hospital. This study was carried out to compare the tooth size and arch dimension of Class I crowded and uncrowded (Class I normal occlusion) malocclusion. The subjects of the study were selected on the basis of inclusion and exclusion criteria. This was a study using 60 pairs of study models divided into two groups (crowded and normal). Each group consisted of 30 pairs of study models of Class I skeletal base and samples were equally divided between the sexes (15 males and 15 females). The subjects were selected from outpatient department of Dhaka Dental College, seeking treatment of malocclusion (Class I crowded group) and students of Dhaka Dental College and Hospital (Class I normal group).

In this study the mean age of crowded group was $15.1 \pm 1.7$ years and the mean age of normal group was $22.5 \pm 1.4$ years. The mean age of this study correlates with the study of Maryam Poosti16 who took the mean age of $16.5 \pm 3.4$ years in the crowded group and $21.3 \pm 2.9$ years in the normal group.

The collective mesiodistal width of six (6) anterior teeth and twelve (12) teeth in the maxilla and the mandible in the crowded group of males and females corroborates with the results of Lundstrom, Fastlicht and Doris et al. who found larger tooth sizes in crowded cases rather than the uncrowded cases, with the greatest difference found between the maxillary lateral incisors and 2 nd premolars.

Present study found both smaller arch dimensions (especially in maxillary width) and greater tooth size in the crowded group compared to the uncrowded groups which coincided with the study of Maryam Poosti.

By evaluating arch dimensions in this study, the most significant difference was seen in maxillary arch width which was greater in the uncrowded group except for the lingual intercanine width which was insignificant. This may be due to buccally placed canines. 
Mandibular arch dimensions, in the transverse plan, did not differ significantly between the uncrowded and crowded groups, except for the lingual intermolar width which was significantly greater in the uncrowded group. This also may be due to racial variation.

On the other hand, the present research suggests a greater tooth size in the crowded group in comparison with the uncrowded one. This result is similar to the result of Maryam Poosti.

\section{CONCLUSION}

The results of this study indicates under two study groups (Class I skeletal base of crowded and uncrowded group)

- Tooth size has a greater contribution to the development of dental crowding.

- Among different arch dimensions maxillary arch width was found to have the most significant difference between two groups.

In conclusion, it may be suggested that Orthodontist who is aware of these differences while evaluating tooth size and arch dimension will be better prepared to diagnose and treatment planning of orthodontic cases more accurately.

\section{REFERENCES:}

1. Proffit WR. Fields HW., Contemporary Orthodontics. 4th ed. St Louis; Mosby; 2001

2. McNamara.AJ., Brudon WL., 1st ed. USA.Neeham press. 2001,p-39.

3. Sperry TP, Worms FW, Isaacson RJ,et al. Tooth size discrepancy in mandibular prognathism. Am. J Orthod.1977 Aug ,72(2), 183-90.

4. Moorees C.F.A, Reed R.B(1964), Correlation among crown diameters of human teeth. Arch. Oral Biol. 9,685-697.

5. Graber TM , Vanarsdall IR ., Orthodontics current principles and techniques 3rd ed. St Louis, Mosby, 2000.
6. Moyers RE (1983a)., Handbook of Orthodontics, 3rd ed Chicago.London. Year book medical publisher, p-352.

7. Lundstrom A., The etiology of crowding of the teeth (based an studies of twins and on morphological investigations) and its bearing on orthodontic treatment (expansion or extraction ). Tr.European Orthodont Soc. 1951, 176-91.

8. Fastlicht j. ,Crowding of mandibular incisor. Am J Orthod 1970, 58, 156-63.

9. Daris JM, Bernard DW , Kuftinee MM ., A biometric study of tooth size and dental crowding. Am J Orthod 1981, 79, 326-35.

10. Howes AE., A polygon of coronal and basal arch dimensions in the Horizontal plane. Am J Orthod 1954, 40,811.

11. Randzic D., Dental crowding and relationship to mesiodistal crown diameters and arch dimension. Am J Orthod Dentofac Orthop $1988,94,50-56$

12. Forsberg CM., Tooth size and spacing in relation to eruption or Impaction of third molars. Am J Orthod Dentofac Orthop. 1988, 94, $57-61$.

13. Gilmore CA, Little RM., Mandibular incisor dimensions and Crowding . Am J Orthod 1984, 86, 493-502.

14. Hossain M. Z, Hoque M. E, Hoque S., Prevalence of malocclusion and treatment facilities at D.D.C. and H. Bangladesh. Jr of Oral Health, 1994,1,4-6.

15. Akhter.Fatema., Evaluation of different malocclusion and probable treatment based on IOTN (Index of Orthodontic Treatment Need) attending at Dhaka Dental College and Hospital. 2007

16. Poosti. M. , Tooth size and arch dimension in uncrowded versus crowded Class- I malocclusion. Jr of Contemporary Dental Practice,Vol- 8, No. 3 , 2007.

\section{Correspondence :}

\section{Dr. Hasnat Jahan BDS, FCPS}

Lecturer

Dept. of Orthodontics and Dentofacial Orthopedics

Dhaka Dental College and Hospital

Mirpur 14, Dhaka 1206

Mobile : +88 01819649817 